\title{
Modeling Oil Palm Pollinator Dynamics using Deterministic and Agent-Based Approaches. Applications on Fruit Set Estimates. Some Preliminary Results
}

\author{
Y. Dumont ${ }^{1,2,3, *}$ | J.-C. Soulié ${ }^{4,5}$ | F. Michel ${ }^{6}$ \\ ${ }_{2}^{1}$ CIRAD, Umr AMAP, Pretoria, South Africa \\ 2 AMAP, Univ. Montpellier, CIRAD, CNRS, INRA, IRD, Montpellier, France \\ 3 Department of Mathematics and Applied Mathematics, University of Pretoria, South Africa \\ ${ }_{5}^{4}$ CIRAD, RU Recycling \& Risk, F-34398 Montpellier, France. \\ 5 Recycling \& Risk, Univ. Montpellier, CIRAD, Montpellier, France \\ ${ }^{6}$ LIRMM, CNRS, University of Montpellier, Montpellier, France \\ *Correspondence: Yves Dumont. Email: yves.dumont@cirad.fr
}

\section{Summary}

Oil palm production is of economical importance in several southern countries. The increasing demand of oil palm put a lot of pressure in several places where the rain forest and thus the tropical diversity is in danger due to deforestation. With the land already cultivated, we need to improve the yields, which means to increase the production of fruits in plots. One way is to increase the pollination or the fruit set through pollination. Palm tree has a specific entomophilous pollinator, a weevil, Elaeidobius spp, that absolutely needs male inflorescence to complete its lifecycle. In young plots (3-7 years old), mainly female inflorescences are produced and thus the pollinator population cannot maintain, resulting in a bad fruit set, and, thus, a bad production. That is why several questions arise: What is the mean number of male inflorescences (per ha) needed to maintain the pollinators population above a certain threshold? And, in terms of yield, what is the optimal size of the population to reach an optimal fruit set? We propose, compare and discuss two different modeling approaches to develop preliminary models to study the dynamics of the Pollinator population, and obtain some rough estimates of the fruit set. We derive some simulations and discuss these preliminary results

KEYWORDS:

Oil Palm; Pollinator; Fruit Set; Metapopulation Model; Agent-Based Modeling; Numerical Simulation

\section{1 | INTRODUCTION}

In the last decades, oil palm (Elaeis guinensis Jacq.) has become a major crops around the World, particularly in South-East Asia and South America, in tropical countries with high rainfall, located around the equator. It originated from West Africa, but was taken to South- and Central America in the 16-17th centuries, and than to the East. Palm oil are extracted from the fruit, that is widely used in food, cosmetics, as well as in the development of biofuel ${ }^{1}$. Compared to other oil crops, oil palm gives the highest yields per hectare (approximately 4 tons/hectare), which explains its rapid expansion. One palm tree produces around $40 \mathrm{~kg}$ of oil palm, according to several exogenous and endogenous parameters, like climatic parameters and pollination. 
Oil palm is monoecious, which means that male and female inflorescences occur separately on the same plant, such that there is almost no self-pollination. When (wild) palm trees are young, they first produce male inflorescences, and, after very few and in proportion that shows little or no regularity. Sometimes inflorescences with both male and female spikelets may occurs, but this is mainly in young palm plantation. This physiology implies a cross-pollination which, in the case of $e$. guineensis, is essentially entomophilous. The action of the pollinator is however conditioned by a moderate-sized palm (young palm tree). When it becomes too tall, usually after 8-9 years, fertilization becomes mainly anemophilous (pollination by the wind).

However, because of increasing demand (up to $2.8 \%$ per year), the cultivated area has increased very fast, impacting places (rain forest) with high biodiversity. In order to maintain/control this expansion, it is mandatory to increase the yields. That is why several oil palm selection programs have been launched in order to increase the production of female inflorescences. Indeed, in optimal growth conditions (e.g. without water stress), selected young oil palm trees produce mainly female inflorescences. Unfortunately, in young palm trees (3-8 years old), pollination is done by natural pollinators, which need male inflorescences, to maintain. To circumvent this difficulty, artificial or manual pollination has been made in South-East Asia until the 80's (and in fact is already used). This was before the work of Rahman Anwar Syed.

Rahman Anwar Syed was a Pakistani entomologist who discovered the natural pollinators of oil palm tree in Cameroon in the late 1970's 234 . His work leaded to the introduction, in the 80's, of E. kamerunicus, the best among the pollinators, thanks to its important pollen carrying capacity ${ }^{4}$. These releases have most often increased the fruit set (percentage of flowers that develop into fruits), that usually depends on pollination ${ }^{1}$, in young palm plantations. However, since the introduction, it has been shown that the maintenance of the population is sometimes difficult (predation by rats, diseases, weather factors, like rainfall, ...), impacting seriously the fruit set.

Our work is a first step towards the complete modeling of palm-pollinator interactions. Thus, we only focus on the weevils population dynamics and the fruit set estimate, using two different, but complementary from our point of view, modeling approaches: Continuous modeling and Agent-Based Modeling (shortly ABM). Using simulations, and comparing both models, we would like to derive some "conditions" to maintain the pollinators population above a certain threshold in order to reach a good fruit set. We also derive pros and cons of both modeling approaches and derive future modeling extensions and knowledge improvements, through observations and experiments. The outline of the paper is as follows: In section 2, we briefly present the interactions between oil palm tree and its main pollinator, $E$. kamerunicus. Based on old results, we derive a rough relationship between the fruit set and the size of the pollinators population. Then, in section 3, we develop the mathematical and the ABM models. Finally, in section 4, we derive several simulations using both models and discuss the results. The paper ends with a conclusion and several perspectives.

\section{2 | ABOUT ELAEIS GUINENSIS AND E. KAMERUNICUS}

\section{1 | About E. Kamerunicus}

Since the pioneering work of Syed, some greenhouse or field works have been done on E. kamerunicus and other Elaeidobius sp. in Cameroon ${ }^{2}$, in Ivory Coast $\frac{56}{6}$, in Malaysia, Indonesia, and India ${ }^{3 / 4778}$. Thanks to these studies, we have some knowledge about the weevil. It has several immature stages ( 1 eggs stage, 3 larvae stages, and 1 pupae stage). On average, a female weevil lays 35 eggs. The whole immature stage is completed within a mean period of $10-14$ days. Its lifespan in the field is estimated around 7 - 10 days. However, we don't know where and when the weevils mate. In addition, weevils can also be impacted by rats, spider webs, insecticides, .... Clearly, along the year, some of its parameters may change, but we do not have any data on that.

E. kamerunicus is really host specific, i.e. it is totally dependent on presence of Elais guinensis male inflorescences. Indeed, the pollinators need male inflorescences to feed and deposit their eggs. According to ${ }^{4}$ and our own experiments, E. kamerunicus seem to have a good dispersal ability. Indeed, they are able to find male (and female) inflorescences, 
because they are attracted by anis odors released by male (and also female) inflorescences in anthesis (mean duration: 5 days for male and 2 days for female). Then, larvae and pupae develop, feeding on decaying flowers. Pollination occurs when weevils visit female inflorescences by accident, attracted by the same aniseed scent as males. Thus clearly, the population size may depend on the available male inflorescences and the fruit set is obviously related to the number of visits in female inflorescences and the density of weevils per female inflorescence. E. kamerunicus was selected and released in the $80^{\prime} \mathrm{s}^{\sqrt[3]{3}}$ because of its dispersal ability and its ability to carry a lot of pollen grains (see also ${ }^{9110[11}$ ).

\section{2 | About Palm Tree, Inflorescence, and Fruit Set}

Palm tree is studied since decades because of its economical importance. Several research institutes, like CIRAD in France, and private companies, like PalmElit, in France, have developed an important "Savoir-Faire" in several places around the world to improve the yields while also lowering the environmental impact. Thus, an abundant literature exists on palm tree. The interested reader can read the Corley \& Tinker's book ${ }^{1}$, or the Woittiez et al. review ${ }^{12}$. In the following, we shortly summarized the main components we use in our models.

In a standard planting, an inflorescence flowers approximately every 2 weeks: It is either male or female. However, due to numerous selection, most of the commercial (young) trees have only female inflorescences, such that the density of male inflorescences per hectare can be really low, which may be problematic to maintain the weevil population, and thus have a good fruit set. According to collected field data (personal communication from Tristan Durand-Gasselin, PalmElit), selection between oil palm trees has driven standard trees to produce almost only female inflorescences along the year, at least the first seven/eight years. That is why, in young plantation, the number of male inflorescences is really small. Climate can also have a strong effect on the inflorescences sex (for instance water stress may induce male inflorescences cycles), however we will not take it into account at this stage.

Female inflorescences are of course of main interest for production. Their pollination occurs by deception. Indeed female inflorescences release the same anis odors than the male inflorescences, thus also attracting pollinators carrying pollen grains. So pollination occurs: The larger the number of visits, the better the pollination, the fruit set, and thus the yield. However, according to our knowledge, only male inflorescences are necessary for the pollinator population dynamics. Therefore we will only consider, at this stage, male inflorescences dynamics in both models.

In fact, they are very few studies on the impact of pollinators on fruit set (or bunch set), because they are difficult to conduct. However, useful data have been obtained, indicating very complex interactions between E. kamerunicus and palm tree inflorescences. Indeed, according to $\frac{10}{}$, the number of adults weevils needs to reach at least 20000 individuals to get a fruit set around $60 \%$. However, according to the literature, the weevils population dynamics seem to be very complex. Indeed, Syed ${ }^{3}$ showed that if the weevils population is too large and the number of male inflorescences is low, this can have a negative effect on the population dynamics (competition for resources).

In most plantations, a standard palm tree density is 143 tree/ha, which leads to the well known pattern provided in Fig. 1 page 4 13 : The distance between each tree is $9 \mathrm{~m}$., and between each row is $7.8 \mathrm{~m}$. We will consider this pattern throughout the paper, but it can be adapted to other plantation densities.

\section{3 | MODELING THE POLLINATOR POPULATION}

As presented in section 2, like other insects, E. kamerunicus has different stages: Eggs stage, 3 larvae stages, 1 pupae stage, and finally the adult stage. For the sake of simplicity, $L$ represents all non-adult (immature) stages, while $P$ represents the adult stage or the (potential) pollinator stage. $L(P)$ number of immature (adults) per hectare. Let $0 \leq \mathrm{MI}_{A}(t)$ be the total number of Male Inflorescence in Anthesis per hectare at time $t$. If one female can reach a male inflorescence, then it can deposit $b$ eggs per day as long as the maximal Larvae capacity per $\mathrm{MI}_{A}, \beta$, is not reached, leading to the following daily birth rate per female Pollinator: 


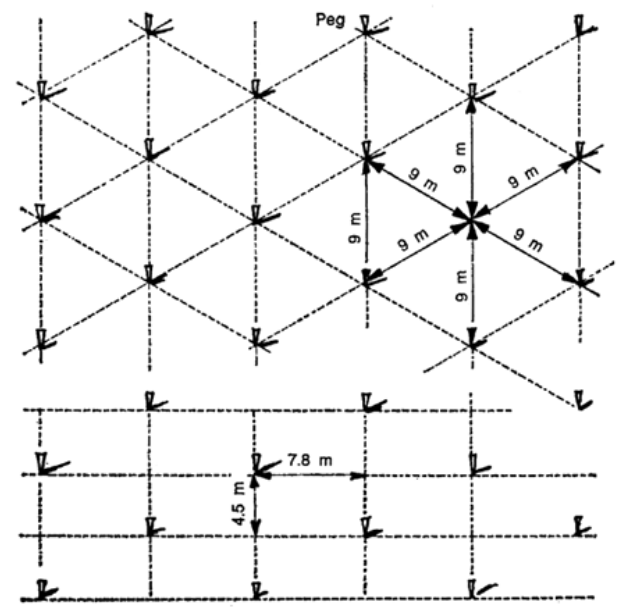

FIGURE 1 Standard planting pattern in oil palm crop: 143 trees per ha

$$
b\left(\mathrm{MI}_{A}(t)-\frac{L}{\beta}\right)
$$

Then, according to the life cycle, after a mean maturation time (in day), $1 / \nu_{L}$, larvae become adults or die at rate $\mu_{L}$. We denote by $\mu_{P}$, the adult mortality rate.

Thus, according to the previous assumptions, we derive the following dynamical system:

$$
\left\{\begin{array}{l}
\frac{d L}{d t}=b r \pi \times\left(\operatorname{MI}_{A}(t)-\frac{L}{\beta}\right) P-\left(\mu_{L}+v_{L}\right) L, \\
\frac{d P}{d t}=v_{L} L-\mu_{P} P,
\end{array}\right.
$$

where $r$ is the sex-ratio, and $\pi$ represents a probability for a pollinator to reach one male inflorescence. We complete the model with initial non-negative conditions, i.e. $L(0) \geq 0$ and $P(0) \geq 0$.

Remark 1. Note here that we only focus on the weevil population dynamics: The male inflorescences dynamics, $\mathrm{MI}_{A}(t)$, may either be given or be an output of an inflorescence dynamic model.

We set

$$
\mathrm{MI}_{A}^{M}=\max _{t} \mathrm{MI}_{A}(t) \quad \text { and } \quad \mathrm{MI}_{A}^{L}=\min _{t} \mathrm{MI}_{A}(t),
$$

and

$$
\mathcal{K}=\left\{(L, P) \in \mathbb{R}_{2}^{+} / L \leq \beta \mathrm{MI}_{A}^{M}, P \leq \frac{\nu_{L} \beta}{\mu_{P}} \mathrm{MI}_{A}^{M}\right\}
$$

Theorem 1. System (1) defines a dissipative dynamical system in the set $\mathcal{K}$.

Proof The right-hand side of system 11 is a continuously differentiable map $\left(C^{1}\right)$. Then, by the Cauchy-Lipschitz theorem, system (1) provides a unique maximal solution. It remains to show that $\mathcal{K}$ is forward-invariant. First, let us note that system 1 , can be rewritten as $\frac{d x}{d x}=A(x) x$, with $A(x)$ being a Metzler Matrix (all off diagonal terms are non-negative) for $x \in \mathbb{R}_{+}^{2}$. Thus, $\mathbb{R}_{+}^{2}$ is invariant by system $[1$, meaning that if $x(0) \geq 0$, then $x(t) \geq 0$, for all time $t>0$. Then, it is straightforward to verify that the compact set $\mathcal{K}$ is an attractive set for system (1), which implies that system (1) is dissipative.

Let us first assume that $\mathrm{MI}_{A}(t)$ is equal to a positive constant, $\mathrm{MI}_{C}$. Then, setting $\mathcal{N}=\frac{b r \pi v_{L} \mathrm{MI}_{C}}{\mu_{P}\left(v_{L}+\mu_{L}\right)}$, and following ${ }^{[14}$ Proposition 1 , we have: 
Theorem 2. Assume $\mathrm{MI}_{A}(t) \equiv \mathrm{MI}_{C}$ is a positive constant, and $\mathcal{N}>1$, then system 11 has a unique positive equilibrium that is Globally Asymptotically Stable (GAS).

Assume now that $\mathrm{MI}_{A}^{L}>0$, then we have:

Theorem 3. If

then system (1) is permanent.

$$
\mathcal{R}=\frac{b r v_{L} \pi \mathrm{MI}_{A}^{L}}{\mu_{P}\left(v_{L}+\mu_{L}\right)}>1
$$

Proof If we consider the following system:

$$
\left\{\begin{array}{l}
\frac{d L_{s}}{d t}=b r \pi \times\left(\mathrm{MI}_{A}^{L}-\frac{L_{s}}{\beta}\right) P_{s}-\left(\mu_{L}+v_{L}\right) L_{s}, \\
\frac{d P_{s}}{d t}=v_{L} L_{s}-\mu_{P} P_{s} .
\end{array}\right.
$$

In fact, when $\mathcal{R}>1$, model (2) admits a unique positive solution $E^{*}=\left(L^{*}, P^{*}\right)$ that is LAS. Bendixson's criterion shows that there is no limit cycles, and, thus according to Poincaré-Bendixson, global stability of $E^{*}$ follows such that if $P_{s}(0)>$ 0 or $L_{s}(0)>0$ then $\left(L_{s}(t)>0\right.$ and $P_{s}(t)>0$ for all $t>0$. In fact, using standard comparison arguments, $\left(L_{s}, P_{s}\right)$ is a lower solution of model $\left\{1\right.$, such that there exists a positive constant $\epsilon$ such that $\min \left\{\lim \inf _{t \rightarrow+\infty} L(t), \lim \inf _{t \rightarrow+\infty} P(t)\right\} \geq \epsilon$.

Theorem 4. If

$$
\mathcal{R}_{M}=\frac{b r v_{L} \pi \mathrm{MI}_{A}^{M}}{\mu_{P}\left(v_{L}+\mu_{L}\right)}<1
$$

then every solution of system (1) converge globally asymptotically to the trivial equilibrium $\mathbf{0}$.

Proof It suffices to consider the following Lyapunov function $V(t)=L(t)+\frac{v_{L}+\mu_{L}}{v_{L}} P(t)$, such that at $\mathbf{0}, V=0$, and $\dot{V} \leq\left(b r \pi \mathrm{MI}_{A}^{M}-\frac{\mu_{P}\left(\mu_{L}+v_{L}\right)}{v_{L}}\right) P-b r \pi \frac{L P}{\beta}=\frac{\mu_{P}\left(\mu_{L}+v_{L}\right)}{v_{L}}\left(\mathcal{R}_{M}-1\right) P-b r \pi \frac{L P}{\beta}<0$, for all $(L, P)$, except $(0,0)$.

Let us now consider $\mathrm{MI}_{A}(t)$ is periodic over a certain period of time, $T>0$, and set

$$
\overline{M I}_{A}=\frac{1}{T} \int_{0}^{T} \mathrm{MI}_{A}(t) d t
$$

In fact $\overline{M I}_{A}$ represents the mean value of MIA, over the period $T$. Then, assuming $2 \mu_{P}>v_{L}$, it is possible to show

Theorem 5. Assume

$$
\overline{M I}_{A}<\frac{\min 2 \mu_{L}+v_{L}, 2 \mu_{P}-v_{L}}{b r \pi}
$$

holds, then every solution of system (1) converge globally asymptotically to the trivial equilibrium $\mathbf{0 .}$

Proof We consider the following functional $V(t)=\frac{1}{2}\left(L^{2}+P^{2}\right)$ that is always positive except at the trivial equilibrium. Computing $\dot{V}$, we derive

$$
\dot{V}=\left(b r \pi\left(\mathrm{MI}_{A}(t)-\frac{L}{\beta}\right)\right) P L-\left(v_{L}+\mu_{L}\right) L^{2}+v_{L} P L-\mu_{P} P^{2} .
$$

Then, using the standard inequalities $P L \leq \frac{1}{2}\left(L^{2}+P^{2}\right)$, and using the fact that $P$ and $L$ are non-negative, we obtain

leading to

$$
\dot{V} \leq\left(b r \pi \mathrm{MI}_{A}(t)\right) \frac{L^{2}+P^{2}}{2}-\left(v_{L}+\mu_{L}\right) L^{2}+v_{L} \frac{L^{2}+P^{2}}{2}-\mu_{P} P^{2},
$$

$$
\dot{V} \leq\left(b r \pi \mathrm{MI}_{A}(t)\right) \frac{L^{2}+P^{2}}{2}-\left(v_{L}+2 \mu_{L}\right) \frac{L^{2}}{2}-\left(2 \mu_{P}-v_{L}\right) \frac{P^{2}}{2}
$$


that is

$$
\dot{V} \leq\left(\operatorname{br} \pi \mathrm{MI}_{A}(t)-\min \left\{v_{L}+2 \mu_{L}, 2 \mu_{P}-v_{L}\right) V .\right.
$$

Solving this inequality leads to $V(t) \leq V(0) e^{\int_{0}^{t}\left(\operatorname{br} \pi \mathrm{MI}_{A}(\tau)-\min \left\{v_{L}+2 \mu_{L}, 2 \mu_{P}-v_{L}\right) d \tau\right.}$. Since, it is well known that for a periodic or a constant function $f$, we have

$$
\lim _{t \rightarrow \infty} \frac{1}{t} \int_{0}^{t} f(\tau) d \tau=\frac{1}{T} \int_{0}^{T} f(\tau) d \tau .
$$

Thus, we deduce that if (3) is verified, then $V(t) \rightarrow 0$ as $t \rightarrow \infty$, which implies that the trivial equilibrium 0 is GAS.

Even if model (1) is conceptually helpful to describe the biological system, it relies on the fact that larvae, pollinators and male inflorescences are homogeneously distributed, which is not the case. Indeed, oil palm trees are distributed as shown in Fig. 1 page 4 and therefore, in some sense, the spatial component needs to be taken into account.

\section{1 | The $n$-Patch Model}

The weevils population dynamics strongly depends on the male inflorescences dynamics that are distributed more or less randomly over space and time. As soon as a male inflorescence emerges and enters in anthesis, it becomes a breeding site, participating to the population emergence and maintenance in the area. To take that into account, we consider a metapopulation model ${ }^{15}$, also called a $n$-Patch model or a Patchy Model (see Fig. 2 page 6), where each palm tree $i$ is a patch, with a sub-population of weevils, $\left(L_{i}, P_{i}\right)$, and $i \in J=\{1, \ldots, n\}$ ( $n$ being the total number of palm trees per ha).

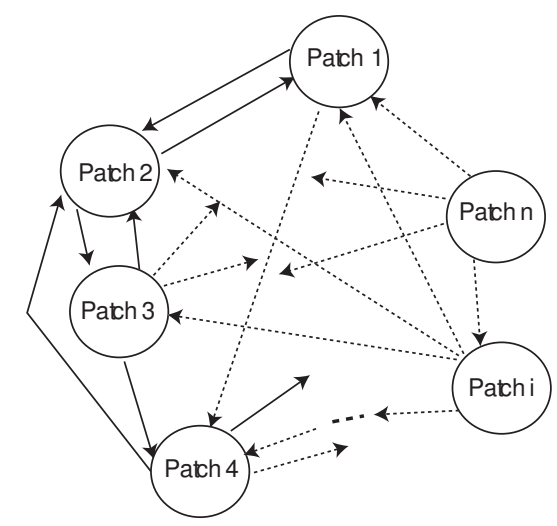

FIGURE 2 A general $n$-Patch model

According to the previous section, for each palm tree $i$, we may have a male inflorescence $\mathrm{MI}_{A, i}$, in anthesis every 13-15 days, following a random process (taking into account that more female inflorescences emerge than male inflorescences on commercial trees in order to improve bunches/fruits production). When $\mathrm{MI}_{A, i}>0$, then pollinators, $P_{j}$, that have emerged in palm tree $j$, may be attracted by $\mathrm{MI}_{A, i}$, depending on the distance between tree $j$ and tree $i$. We will assume that the "attractivity" function, $f$, is decreasing according to $d_{i j}$, the distance between $i$ and $j$, and increasing according to $\lambda>0$ that represents the dispersal ability of the weevil (note that we do not make a difference between male and female pollinator). In other words, pollinators that are far from $I M_{A, i}$ have little chance to find $\mathrm{MI}_{A, i}$ and thus breed. Altogether, the probability for pollinator $P_{j}$ to find $\mathrm{MI}_{A, i}$ can be modeled by $f\left(\lambda, d_{i j}\right)=e^{-d_{i j} / \lambda}$, for instance. 
Of course among the pollinators that are attracted by the inflorescence in anthesis, only female can lay eggs, with a proportion $r$. Thus, following model (1), the growth rate is modelled by

$$
\operatorname{br} \sum_{j=1, j \neq i}^{n} f\left(\lambda, d_{i j}\right) P_{j} \times\left(1-\frac{L_{i}}{\beta_{i}}\right),
$$

where $n$ is the number of palm trees per hectare. Here, we assume that $\beta_{i} \in\left[\beta_{\min }, \beta_{\max }\right]$, where $0<\beta_{\min }$ and $\beta_{\max }$ are the minimal and maximal larvae capacity of a male inflorescence in palm tree $i$. It is important to understand that pollinators emerging from inflorescence $i$ will not be able to breed in the same inflorescence because they spread away immediately after emergence, and the anthesis stage is limited in time (5 days) and maturation time for eggs and larvae is beyond this anthesis stage. Last but not least, it is highly improbable to have 2 consecutive male inflorescences on tree $i$, at least in standard growth condition. Altogether, the Patchy-Model for the weevil population becomes

$$
\left\{\begin{aligned}
\frac{d L_{i}}{d t}= & b r \sum_{j=1, j \neq i}^{n} f\left(\lambda, d_{i j}\right) P_{j} \times\left(\mathrm{MI}_{A, i}(t)-\frac{L_{i}}{\beta_{i}}\right) \\
& -\left(\mu_{L}+v_{L}\right) L_{i}, \\
\frac{d P_{i}}{d t}= & v_{L} L_{i}-\mu_{P} P_{i},
\end{aligned}\right.
$$

for $i=1, \ldots, n$.

The time-dependent parameter $\mathrm{MI}_{A, i}(t)$ comes either from field data or as an output from an other model. We know that the male inflorescence anthesis lasts 5 days, with an increasing attractivity the first 2-3 days, and a decaying until day 5. We also assume that $\mathrm{MI}_{A}(t)$ is sufficiently smooth in order to apply standard mathematical results. According to 4, and setting $L=\left(L_{1}, \ldots, L_{n}\right), P=\left(P_{1}, \ldots, P_{n}\right)$, we have

$$
\left\{\begin{array}{l}
\frac{d L}{d t}=\mathcal{M}_{P} P-D(P) L \\
\frac{d P}{d t}=v_{L} L-\mu_{P} P
\end{array}\right.
$$

where $\mathcal{D}$ is a diagonal matrix

$$
(\mathcal{D})_{i, j}(P)= \begin{cases}\mu_{L}+v_{L}+b r \sum_{j=1, j \neq i}^{n} f\left(\lambda, d_{i j}\right) \frac{P_{j}}{\beta_{i}} & j=i, \\ 0 & j \neq i\end{cases}
$$

and $\mathcal{M}_{p}$ is a non-negative matrix such that

$$
\left(\mathcal{M}_{P}\right)_{i, j}= \begin{cases}\mathrm{MI}_{A, i}(t) \times \operatorname{brf}\left(\lambda, d_{i j}\right) & j \neq i, \\ 0 & j=i\end{cases}
$$

We deduce that system (5) is positively invariant, which means that any trajectory starting in the positive orthant $\mathbb{R}_{+}^{n} \times \mathbb{R}_{+}^{n}$ remains forever in $\mathbb{R}_{+}^{n} \times \mathbb{R}_{+}^{n}$. Thus biologically our system is meaningful. Note also that the right-hand side of system (5) being Lipschitz continuous, system (5) admits a unique solution. Setting

$$
\mathcal{K}_{i}=\left\{\left(L_{i}, P_{i}\right) \in \mathbb{R}_{2}^{+} / L \leq \beta_{i} \mathrm{MI}_{A, i, \max }, P \leq \frac{\nu_{L} \beta_{i}}{\mu_{P}} \mathrm{MI}_{A, i, \max }\right\},
$$

it is straightforward to show that $\prod_{i=1}^{n} \mathcal{K}_{i}$ is an absorbing set. Thus the solution of system (5) is bounded. Setting $\mathbf{L}=\sum_{i=1}^{n} L_{i}$ and $\mathbf{P}=\sum_{i=1}^{n} P_{i}$, we derive the following system

$$
\left\{\begin{array}{l}
\frac{d \mathbf{L}}{d t}=b r \sum_{i=1}^{n}\left(\sum_{j=1, j \neq i}^{n} f\left(\lambda, d_{i j}\right) P_{j}\right) \times\left(\mathrm{MI}_{A, i}(t)-\frac{L_{i}}{\beta_{i}}\right)-\left(\mu_{L}+v_{L}\right) \mathbf{L}, \\
\frac{d \mathbf{P}}{d t}=v_{L} \mathbf{L}-\mu_{P} \mathbf{P} .
\end{array}\right.
$$


Then, we assume that whatever $\lambda>0$ and whatever $i$ and $j$ in $J$, there exists $d_{\min }$ and $d_{\max }$ such that $f_{\min }=f\left(\lambda, d_{\min }\right) \leq$ $f\left(\lambda, d_{i j}\right) \leq f_{\max }=f\left(\lambda, d_{\max }\right)$ such that

$$
\sum_{i=1}^{n}\left(\sum_{j=1, j \neq i}^{n} f\left(\lambda, d_{i j}\right) P_{j}\right) \mathrm{MI}_{A, i}(t) \geq\left(\sum_{i=1}^{n} \mathrm{MI}_{A, i}(t)\right) f_{\min } \mathbf{P}
$$

and

$$
\sum_{i=1}^{n}\left(\sum_{j=1, j \neq i}^{n} f\left(\lambda, d_{i j}\right) P_{j}\right) \frac{L_{i}}{\beta_{i}} \leq \frac{f_{\max }}{\beta} \mathbf{P} \mathbf{L},
$$

where $\beta \leq \beta_{i}$. From [6, we deduce that

$$
\left\{\begin{array}{l}
\frac{d \mathbf{L}}{d t}=b r\left(\left(\sum_{i=1}^{n} \mathrm{MI}_{A, i}(t)\right) f_{\min }-\frac{f_{\max } \mathbf{L}}{\beta}\right) \mathbf{P}-\left(\mu_{L}+v_{L}\right) \mathbf{L}, \\
\frac{d \mathbf{P}}{d t}=v_{L} \mathbf{L}-\mu_{P} \mathbf{P} .
\end{array}\right.
$$

Thus, assuming that $\sum_{i=1}^{n} I M_{A, i}(t) \geq I M_{A}>0$ for all $t>0$, and applying Theorem 3 page 5 . we deduce that system (6) is permanent if

$$
\mathcal{R}_{\text {patch }}=\frac{b r \mathrm{MI}_{A} f_{\min } v_{l}}{\left(v_{L}+\mu_{L}\right) \mu_{P}}>1
$$

Thus, when $\mathcal{R}_{\text {patch }}>1$, there exists a positive constant $\epsilon_{p}$ such that

$$
\min \left\{\lim \inf _{t \rightarrow+\infty} L(t), \lim \inf _{t \rightarrow+\infty} P(t)\right\} \geq \epsilon_{p} .
$$

Let us emphasize that we only here focus on the population establishment in the whole plot and not on each patch. Indeed, since the anthesis stage is limited in time, the birth term in each patch is positive only during this period of time. After 2 weeks of development, the young pollinators will leave their inflorescence to seek after another one, in anthesis, to pursuit the cycle.

Remark 2. Let us also notice that the choice of $f\left(\lambda, d_{i j}\right)=e^{-d_{i j} / \lambda}$ is not the only choice. Indeed, even if $d_{i j}$ is large, $e^{-d_{i j} / \lambda}>0$, which is not always realistic. If we consider a larger plot (more than one hectare for instance), the connection between palm tree $i$ and $j$ is not always possible. Thus, we may choose a function such that only a palm tree localized at a certain distance from palm tree $i$ can be reached (in a day) by pollinators emerging from palm tree $i$. Thus, if we consider that $d_{\text {max }}$ is the maximum distance that pollinators can travel to reach palm tree $i$, such that the function $f$ can be chosen as follows:

$$
f\left(\lambda, d_{i j}\right)=\lambda \pi \max \left\{1-\frac{d_{i j}}{d_{\max }}, 0\right\}
$$

where $\pi \max \left\{1-\frac{d_{i j}}{d_{\max }}, 0\right\}$ can be seen as the probability for a pollinator to find inflorescence $i$ from inflorescence $j$.

In fact, it is not only important to show that it is possible to maintain the pollinators population, but also to reach another threshold, interesting from the pollination or the fruit set point of view. This will be investigated in the subsection dedicated to the numerical simulations.

\section{2 | The Agent-Based Modeling Approach}

By focusing on local rather than global interactions, Agent-Based Modeling (ABM) appears as an interesting and complementary approach for studying complex systems ${ }^{17}$. Especially, ABM is a kind of Individual Based Modeling (IBM) that not only relies on representing the individuals but also their concrete behaviors, actions, interactions and physical environment. Therefore, it allows the modeling of relatively complex systems while keeping with a simulation approach which makes it possible to explicitly represent and analyze the dynamics of these systems, both globally and with a fairly high degree of detail thanks to the modeling of micro-level interactions. So, ABMs can be seen as artificial 
laboratories wherein it is possible to directly modify the behaviors and interactions of the individuals to observe their influences on the global dynamics of the studied system 18 .

In the scope of this research, one major interest of using an ABM relies on the fact that it allows to both model different individuals and concretely represent the moving of the weevils within the considered parcel, and thus the time required for each individual to move from one site to another. Therefore, this allows to study the influence of microscopic aspects on the global dynamics. Especially, we are interested in modeling (1) how the weevils are individually attracted to palm trees with a male inflorescence, (2) how they explore the parcel (which may lead to leave it), (3) how they individually pollinate a palm tree, and (4) the dispersal ability of each weevil. Moreover, it is also easy to integrate different behaviors into the modeling for distinguishing male and female pollinators, and thus their mutual interactions.

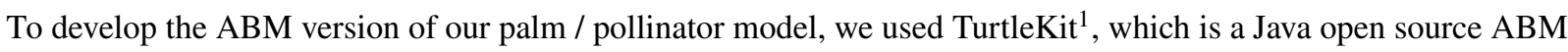
platform ${ }^{19}$ dedicated to spatialized ABM simulations. Like the popular NetLogo platform ${ }^{20}$, TurtleKit is based on the very intuitive Logo programming approach ${ }^{21}$. However, the TurtleKit's programming interface is open and sticks to the Java's syntax and ecosystem so that its simulation engine can be easily extended and customized, thus allowing the required optimization for simulating dozens of thousands of agents, as it is the case in this research. Notably, TurtleKit is designed to allow the use of the General-Purpose computing on Graphics Processing Units (GPGPU) technology for high performance computing ${ }^{22}$, which will certainly be a critical asset when we will consider the simulation of several hectares at once. Moreover, TurtleKit is based on the Agent/Group/Role (AGR) paradigm ${ }^{[23}$, which is an organizationbased model for defining the structure of multi-agent systems and their internal interaction schemes. AGR relies on three main concepts:

- Agent: An agent is an active, communicating entity playing roles within groups. An agent may hold multiple roles, and may be member of several groups;

- Group: A group is a set of agents sharing some common characteristic. Two agents may communicate if and only if they belong to the same group, but an agent may belong to several groups;

- Role: The role is the abstract representation of a functional position of an agent in a group. An agent must play a role in a group, but an agent may play several roles. And a role may be played by several agents.

In the scope of our modeling, roles are used to both easily distinguish the individuals with respect to their specificity and specialize their behaviors accordingly. So, in our project we have defined three types of agents: Palm, Pollinator and Larva.

A Palm agent represents a palm tree within a palm plantation. The palm trees are located within a spatial environment (a $100 m \times 100 m$ square) representing the crop, according to Figure 1 The behavior of a palm agent is very simple and only computes its next state on a day by day basis, thus implementing the lifecycle which has been previously defined. So a palm agent may play the following roles:

- growth: When the palm tree is growing. The duration of this role is defined by a parameter called TBIA, which stands for Time Between Inflorescences in Anthesis. When this duration is reached, the palm tree switches its role to either male (inflorescence) or female according to a sex ratio, namely MIR (Male Inflorescence Ratio), a parameter which is set to 0.05 or 0.1 according to different scenarios;

- male: The duration of this role is fixed to 5 days for now. When playing this role, the palm tree attracts the weevils according to the distance chosen for the Male Inflorescence Attraction Radius (MIAR), i.e. $r_{M A S}$ in Table 2 When the duration of this role is reached, the palm tree switches back to the growth role;

- female: The duration of this role is fixed to 2 days. The palm tree does not attract the weevils when playing this role. When this period ends, the palm tree plays the growth role again. 
The Pollinator agent represents a single weevil and plays only either the male role or the female role. Pollinator agents have a more complex behavioral model since it relies on several atomic behaviors which can be triggered according to the role they play:

- looking: This behavior is used when the Pollinator is looking for a male palm tree. When it detects one, it goes directly to it. When the Pollinator reaches its target, it then either triggers the pollinate behavior (for female) or feed (for male). Note that the target may change dynamically according to the palm tree life-cycle;

- explore: The Pollinator explores only the space around it without feeling the need to find a palm tree. It may therefore leave the parcel and thus the simulation as well;

- feed (only male): The purpose of this behavior is only to model that a male pollinator stays a while on a palm tree;

- pollinate (only female): This behavior models that a female weevil starts to spawn larvae on the male inflorescence, according to a number of larvae per day per female which is set to 3 (parameter $b$ in Table 2 . Also, new larvae can only be added if the maximal larvae capacity of a male inflorescence is not yet reached $\left(\beta=10^{4}\right.$, as defined in Table 2 ;

- dead: This behavior is triggered when Pollinator agents reach the end of their life expectancy, thus leaving the simulation.

Finally, the Larva agent is the simplest one since its behavior only relies on creating a new male or female adult Pollinator when it reaches its maturation time (14 days as defined in Table 2 , according to the parameter implementing the sex-ratio set to 2/3 (i.e. $r$ in Table 2 . Still, a larva may die before reaching the adult stage according to the larvae death rate $\mu_{L}$ (0.05 percent per day). So larva agents play only one single role in the simulation, namely Larva.

\section{4 | SIMULATIONS}

We now derive some simulations for the patchy model and the ABM. Since we are not only interested in the population dynamics but on the relationship with the fruit set, we briefly discuss in the next subsection the data obtained by Syed.

\section{1 | Fruit Set Estimates}

The weevils population dynamics is driven by Model (4). However, we are mainly interested in the fruit set or the bunches production. Of course, several factors can affect the fruit set, however it is clearly stated that E. kamerunicus is essential in palm tree plantation, at least when the plantation is young. In the beginning of the 80 's, after the discovery of the pollinators by Syed, E. kamerunicus has been introduced in Malaysia and Indonesia. As a result, in most places, an increase of the fruit set has been observed during the first years. However, in some places, the fruit set did not increase nor varied so much. Thus, entomologists started to study the relationship between the weevils population and the fruit set, trying to define a pollination force, or at least estimate the number of pollinators allowing a good fruit set or even reaching the optimum fruit set threshold, i.e. $70 \%$.

$\operatorname{In} \frac{10}{10}$, the authors summarized various results about the number of pollinators required to reach a certain fruit set. For instance some authors indicated that at least 20000 weevils are necessary to have a fruit set of $60 \%$. To the best of our knowledge, the most precise work is Syed \& Salleh's ${ }^{24}$, showing that 1500 adult weevils can pollinate about $50 \%$ of fruits, and the optimum fruit set (around 70\% and above) is obtained with 3000 adult weevils per female inflorescence. As far as we know, no other works of this type have been done since then.

Syed \& Salleh's ${ }^{24}$ provides very interesting data between the weevils population per ha and the fruit set. Some of these data are summarized in Fig 3 , page 11 The points represent the fruit sets estimated in different areas under different weevils density per hectare. Unfortunately, we do not have so much details about how these data have been recorded. 
The blue curve in Fig 3 , page 11 is a nonlinear approximation of the potential fruit set according to the weevil population size per hectare, following

$$
F S(t) \approx a \log _{10}(P(t)),
$$

where $a=0.1491$ and a 95\% confidence interval [0.1401451, 0.1579961]. This is a rough relationship. However, it seems clear that a mean density above 20000 weevils per ha is necessary to have a fruit set above $60 \%$, as claimed in the literature. We will use (9) in the numerical simulations to estimate the fruit set and its variation with respect to the time, for each population estimate, and for discussions.

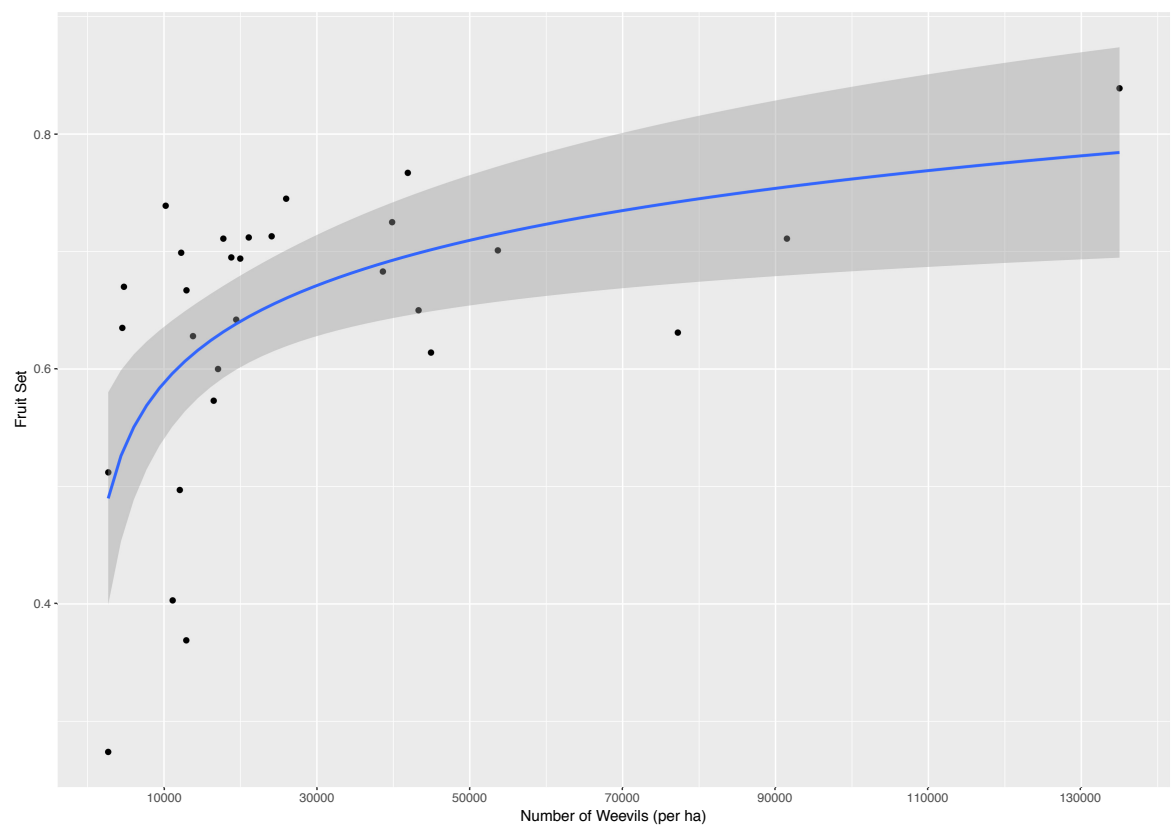

FIGURE 3 Relationship between the fruit set and the mean number of weevils per ha, based on field data ${ }^{24}$. The gray area represents the $95 \%$ confidence interval.

\subsection{Simulations with the Patchy Odes Model}

Simulations have been carried out using a MacBook Pro with 16Gb Memory and a 2,2 GHz Intel Core i7. Parameters values used to setup simulations are given in Table 2 page 20 We first consider simulations with $f\left(\lambda, d_{i j}\right)=e^{-d_{i j} / \lambda}$ and then with $f$ as defined in $(8)$, page 8

We need additional data related to MIA. We build MIA data for each palm tree taking into account that each palm tree produces an inflorescences every 2 weeks (preferably female inflorescences). Thus, we will consider two MIA data (see Fig. 4 page 12): The first one where the daily mean density of MIA is 2.1, case (a), and the second with a daily mean density of 4.4 , case (b).

It is very important to understand that a MIA density $I M_{A}$ means that, in mean, $I M_{A}$ male inflorescences are always in anthesis.

We run simulations for the two MIA densities using the parameters values given in Table 2 , page 20. We also start with an initial population of 100 adults. 

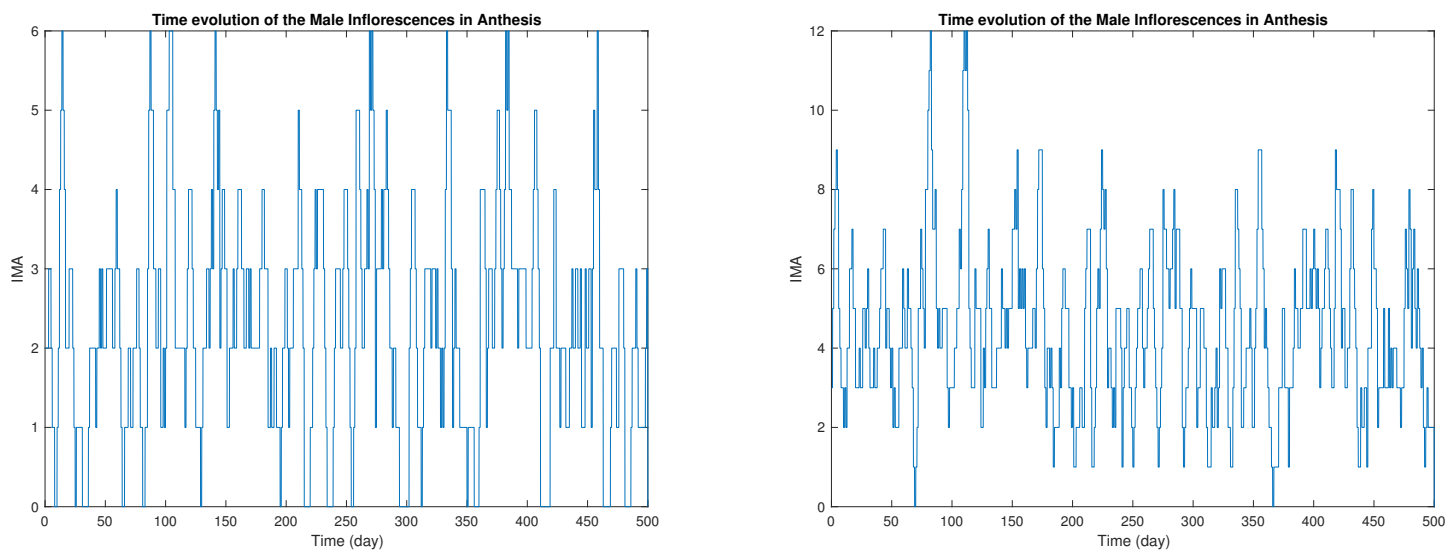

FIGURE 4 Time evolution of MIA per ha, with a mean number equal to (a) 2.1 and (b) 4.2
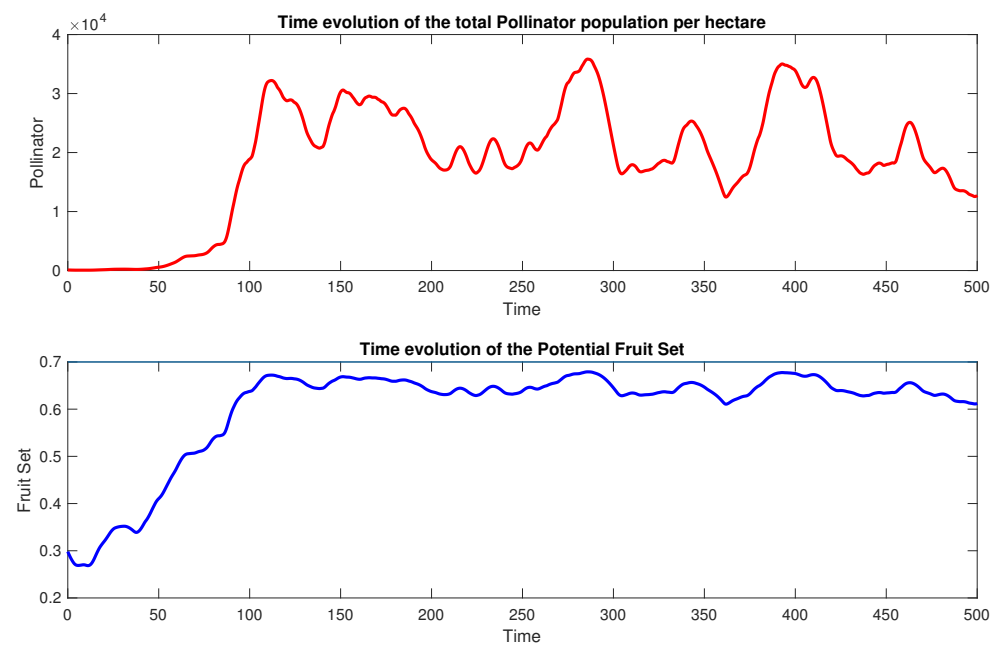

FIGURE 5 Time evolution of (a) E. kamerunicus population on one hectare, (b) the fruit set, when the daily mean density of MIA is 2.1 and $\lambda=20$

As seen in Figs. 5 a) and 6 a), page 13 , the population size varies significantly, according to the number of MIA available for breeding (Fig. 4 . Clearly the mean number of MIA has a great effect on the population size, even if the variability of the latter is important.

It is also interesting to notice the difference in the delay needed for the population to settle. When the MIA density is small, then the population needs a longer time to settle and then increase, while this is much faster when the MIA is greater than 4 . However, the settlement and the population size may also depend on other parameters, such as $\lambda$.

In fact, using the relationship between the fruit set and the population size per ha, Figs. 5 b) and 6 (b), page 13 . show clearly that large amplitudes in the population size do not impact too much the fruit set variation. In fact, once the population is large enough, even with large variation, the fruit set can reach and maintain around the threshold of $70 \%$. This agrees well with the literature. However, data are needed to validate these preliminary results. 

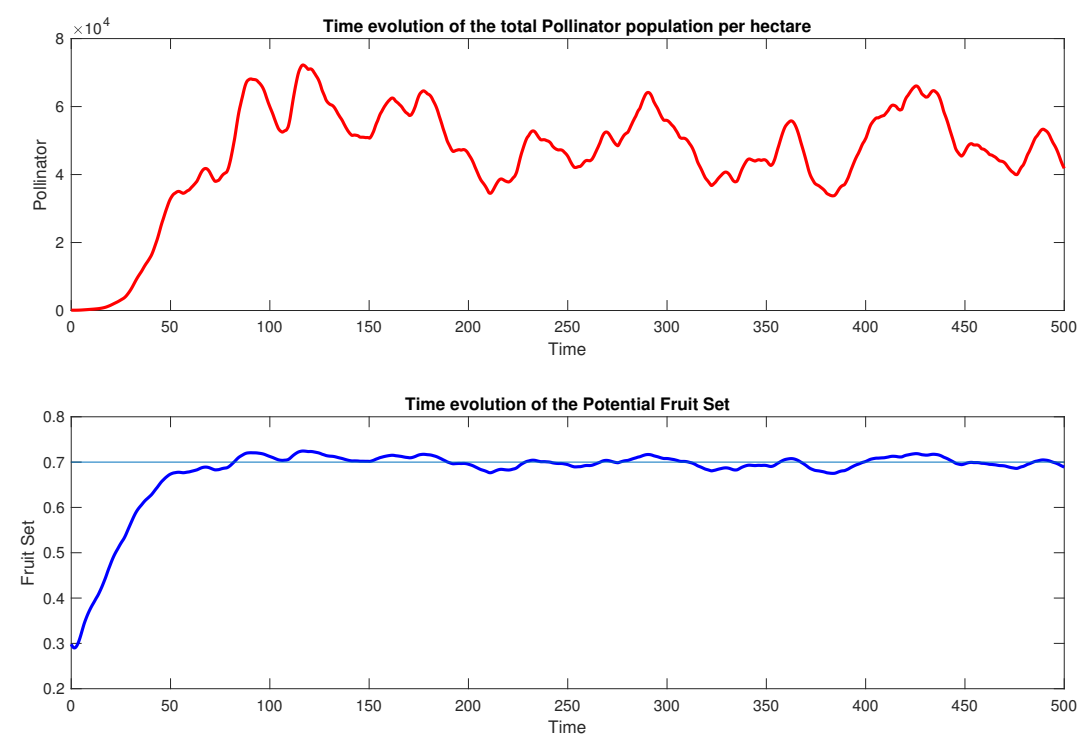

FIGURE 6 Time evolution of (a) E. kamerunicus population on one hectare, (b) the fruit set, when the daily mean density of MIA is 4.2 and $\lambda=20$
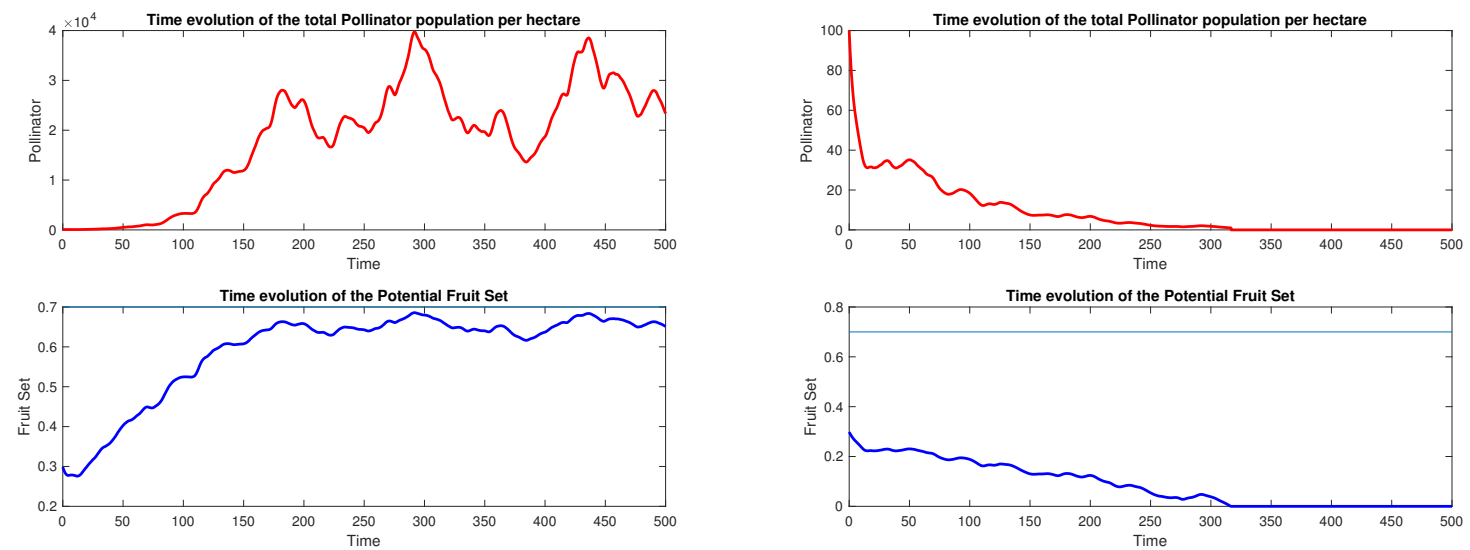

FIGURE 7 Time evolution of (a) E. kamerunicus population on one hectare, (b) the fruit set, when the daily mean density of MIA is 4.2. Two cases: $\lambda=\frac{20}{2}=10$ and $\lambda=\frac{20}{3}$

Fig. 5 b), page 12 with $\lambda=20$, shows clearly that the weevils population is not sufficient to reach the optimal fruit set. In fact, with a mean daily number of MIA around 2.1, a fruit set around 0.6-0.65 can be reached.In Fig. 6 b), page 13. we clearly show that when the mean number of MIA is between $4-5$, the optimal fruit set, $70 \%$, can be reached.

Of course, these simulations take place in an "optimal" environment (without heavy rainfall, predation on the larvae population, ....).

If for instance, we take into account additional events that may disturb the weevils spreading (like female inflorescences in anthesis, heavy rainfall, ...), these may impact $\lambda>0$ and/or $d_{\max }$ : A small $\lambda$ implies a bad dispersal ability such that the emerging weevils are not necessarily able to find new MIA to deposit their eggs. As example, if we consider $\lambda=\frac{20}{2}=10$ or $\lambda=\frac{20}{3}$, we show in Fig. 7 page 13 , that this may impact the population dynamics: The population 
either settle or disappear. Consequently, $\lambda$ is an important parameter that needs further investigations for estimating its interval of values, under different weather and/or natural conditions. It is now interesting to provide the same simulations,
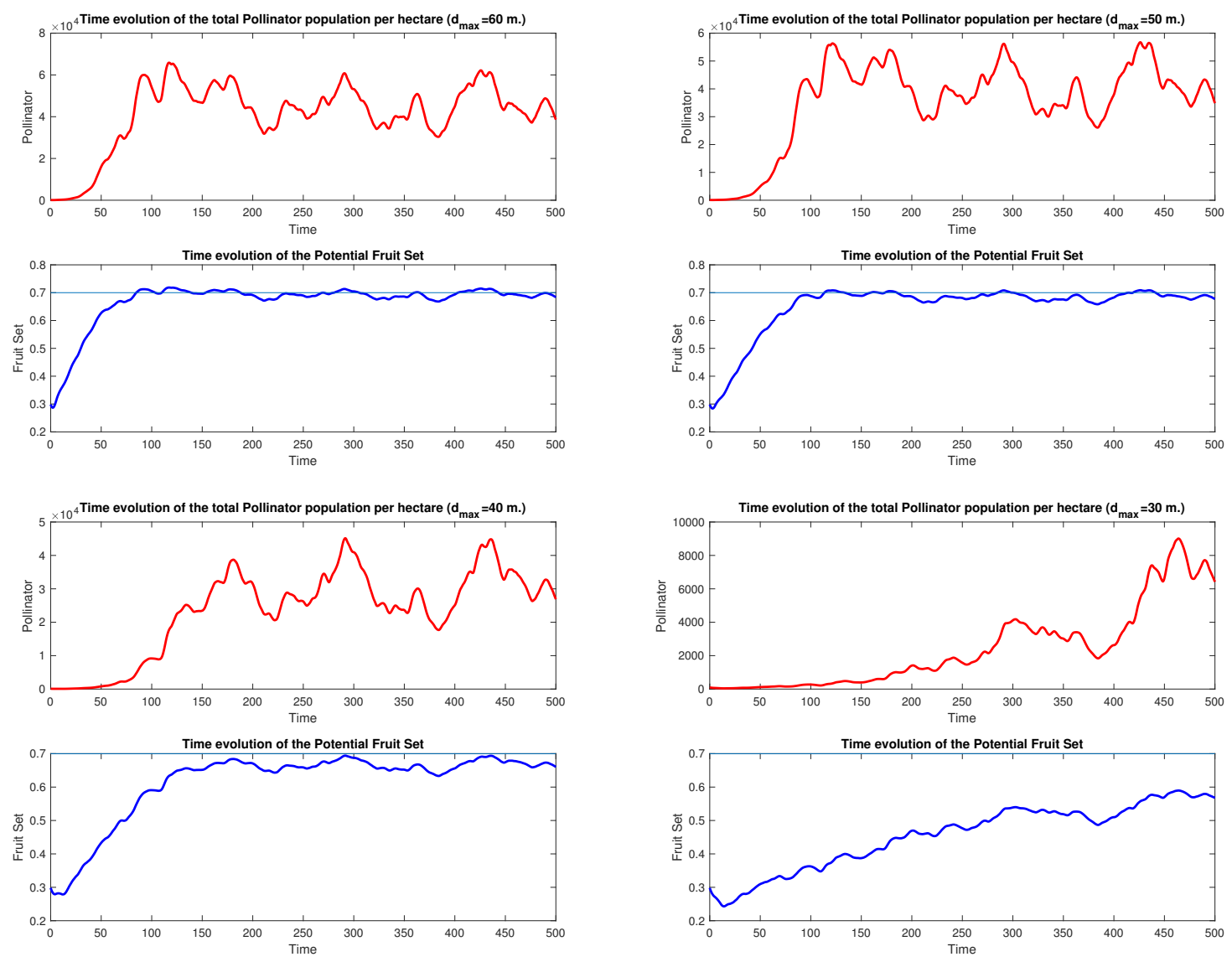

FIGURE 8 Time evolution of E. kamerunicus population (per ha), the fruit set, when the daily mean density of MIA is 4.2 , and $\lambda=20$ for different values of $d_{\max }$ : (a) $d_{\max }=60$, (b) $d_{\max }=50$, (c) $d_{\max }=40$, (d) $d_{\max }=30$

but taking into account that all patches are not (directly) connected to each other, i.e. using the attractiveness function defined in 8 , page 8 . In Fig. 8 we show simulations when $\overline{M I A}=4.2, \lambda=20, \pi=0.02$, and various values for $d_{\max }$ : The weevils population is persistent in all cases, but the smaller the value of $d_{\max }$ the lower the number of connected patches. In other words, the matrix $\mathcal{M}_{P}$ is sparse when $d_{\max }$ is small. This coupled with the fact that the mean number of MIA is small, i.e. $\overline{I M_{A}}=2.1$ leads to Fig. 9 page 15 where the weevils population takes a long time to settle and, in fact, is not able to settle when $d_{\max }$ is less than $45 \mathrm{~m}$.

In fact, it is clear that if for any reason the dispersal from one patch to another is limited, the population has less chance to settle or sustain. Thus, obviously, if along the season, $d_{\max }$ varies, then this can have an impact on the population permanence, thus on its size, and finally on the fruit set.

Whatever the function chosen to take into account the dispersal capacity and the weevil's ability to find a MIA, it is clear that the parameters $\lambda, d_{\max }$ and $\pi$ are of utmost importance to estimate. This needs to be done through field experiments.

In the next subsection, we consider an ABM approach that seems to be more "realistic" from the biological point of view, in that sense that each insect will be simulated. At first glance, this approach may be seen opposite to the patchy 

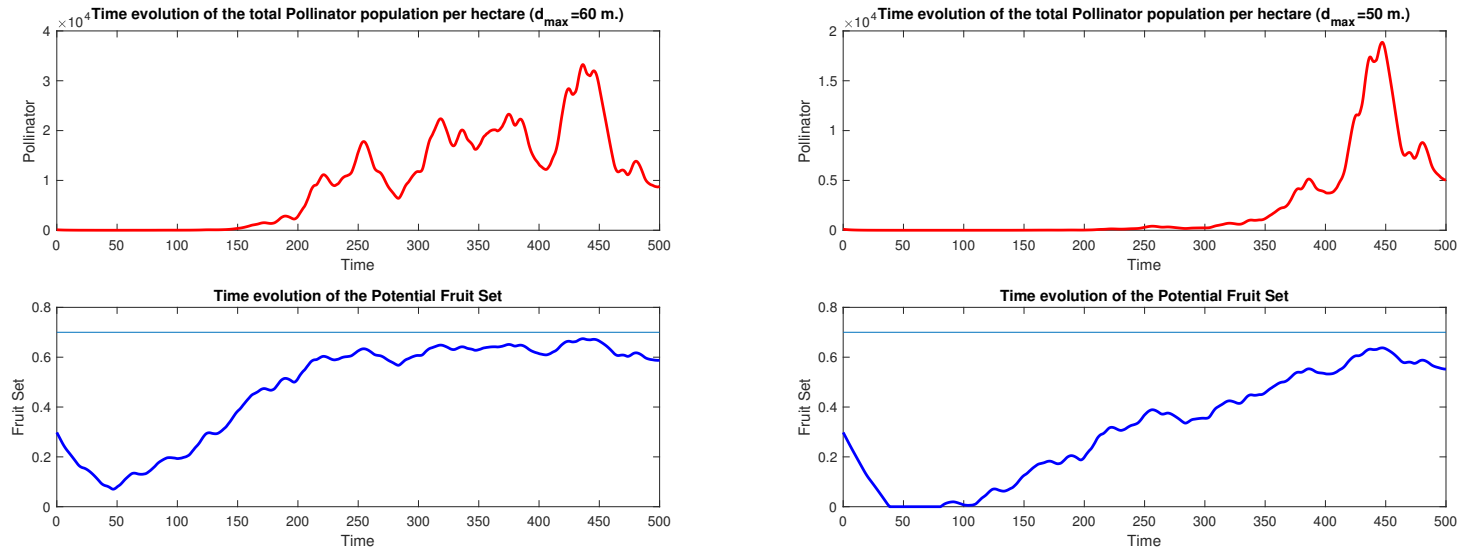

FIGURE 9 Time evolution of E. kamerunicus population (per ha), the fruit set, when the daily mean density of MIA is 2.1 , and $\lambda=20$ for different values of $d_{\max }$ : (a) $d_{\max }=60$, (b) $d_{\max }=50$

model. However, it can be more complicated to develop since the deep understanding of all processes are needed in order to implement all possible behaviours.

\section{3 | Simulations using ABM}

Simulations have been carried out using a workstation with $16 \mathrm{~Gb}$ of memory and a $3.5 \mathrm{GHz}$ Xeon processor. Moreover, we considered the same biological parameters than those used for simulating the deterministic model (that is those given in Table 2 page 20p.

We considered also the same data for the male inflorescence in anthesis (with MIA mean densities of 2.1 and 4.2), and have done about one hundred simulations for each scenario. One run for a 2.1 MIA mean density takes about 5 minutes and around 10 minutes for 4.2. This drastic increase of simulation time is due to the fact that, in the second case, there are more individual weevils thanks to more breeding sites, and consequently the simulator has to manage and compute far more individual behaviours.

In Fig. 10 a), page 16 and Fig. 11 a), page 17, we show the mean behavior obtained with 100 ABM simulations of the weevils population per hectare for each MIA density. Clearly, although the populations maximum size are approximately equivalent, the mean sizes are lower compared to those obtained with the deterministic model, mainly because of a larger amplitude in the population dynamics. This is principally due to the fact that, with an ABM approach, each individual behavior is simulated, which leads to diverse trajectories for all insects, and thus introduces more sensitivity to micro-level conditions. For instance, when there is only one or two male inflorescences available, if a new one takes place far from a crowded breeding site then the population may severely decrease. Of course, these results may depend on different parameters like the range of attraction of the MIA and the dispersal ability of the weevils.

Of course, drastic changes in the pollinators population automatically impact the fruit set, as illustrated in Fig. 10 b), page 16, and Fig. 11 b), page 17 where the variations in fruit set are much more important than those obtained with the deterministic model.

Compared to the fruit set estimate obtained with the deterministic model (see Figs. 5 and 6 , the ABM fruit set estimates are lower than the optimal value 0.7. However, according to field experts, the MIA mean value required to maintain a weevil population, and thus reach the optimal fruit set, should be in $[4, \ldots, 7]$. This is almost confirmed by our numerical simulations. However, we did not take into account all processes and in particular the fact that Female Inflorescences in Anthesis (FIA) can also attract the weevils and thus reduce drastically their spreading to MIA. Of 

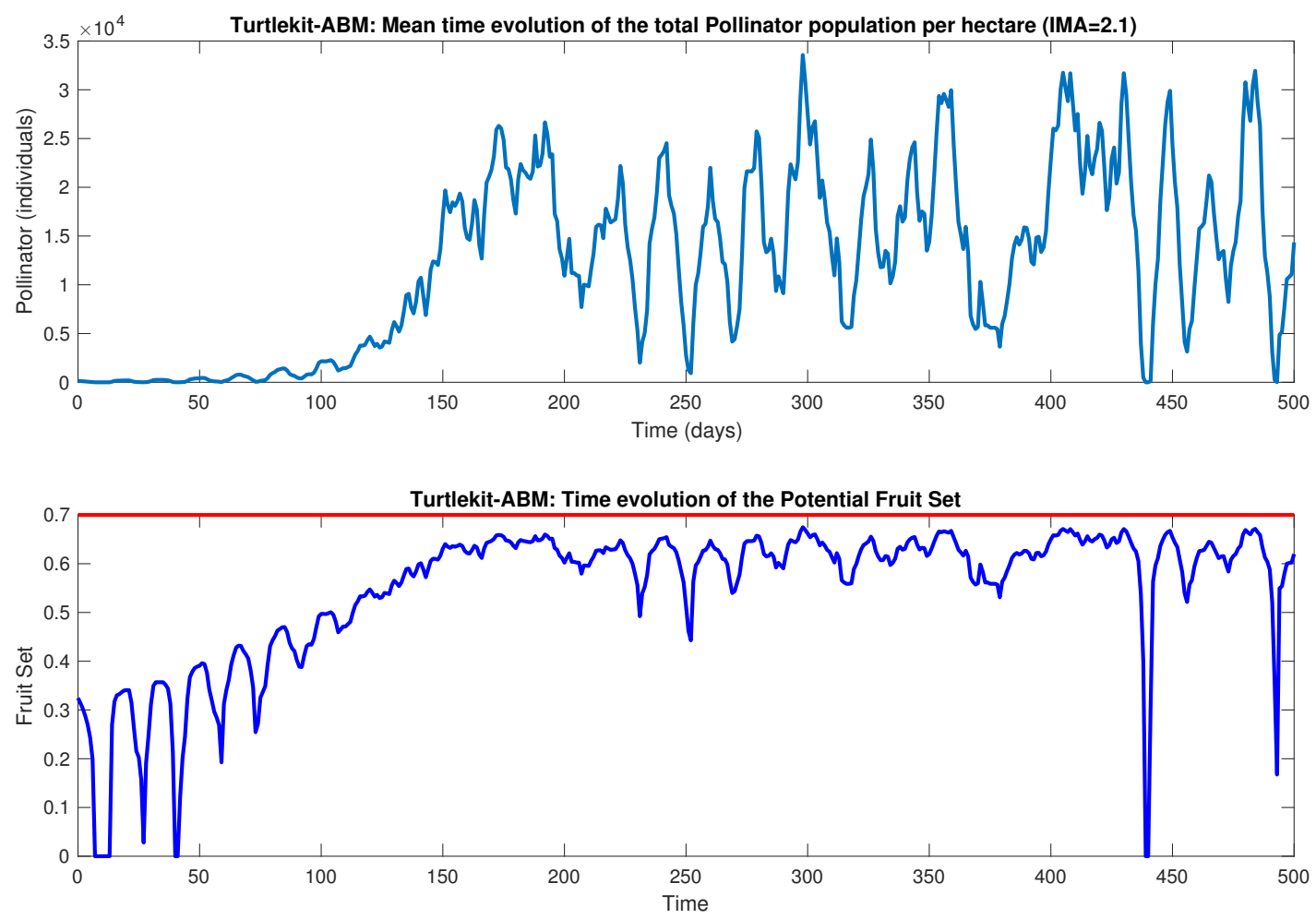

FIGURE 10 ABM simulations. Time evolution of (a) the pollinator population; (b) the potential fruit set when the MIA is 2.1

course, visits in FIA are necessary for pollination and thus fruit set. Finally, several parameters may be related to environmental parameters that may disturb the weevils population.

Although all these simulation results need to be compared with real data, which are really scarce in the (available) literature. Then, we believe that the population size estimate stands between the deterministic and the ABM simulations. The main advantage of the deterministic approach is that simulations are obtained in less than 10 seconds, while it takes several minutes for only one simulation with the ABM. In addition, the mathematical model can be studied theoretically and, eventually, derive useful information on some parameters and/or all possible dynamics. Finally, it could evolved to a stochastic model, taking into account some stochasticity in the parameters, like in the ABM.

\section{4 | Agent-Based Model Sensitivity Analysis}

For gaining more insights on the ABM, we performed a sensitivity analysis based on the extended-Fourier Amplitude Sensitivity Testing (eFAST) ${ }^{25}$ method which we used in the context of the R software, i.e. by using the fast 99 function of the sensitivity package of the $\mathrm{R}$ environment. One the main advantage of this method is that it allows the estimation of first order and total Sobol indices for all the factors ( $p$ indices and each with a set of $n$ values) at a total cost of $n \times p$ simulations. As a consequence, this method decreases drastically the simulations number and thus the computation time. Here follows the description of the experimental settings used for this analysis.

FAST relies on specifying the bounds of the different parameters (factors). Those are used for computing the factors' values to be considered for each experiment by discretizing the corresponding intervals according to particular sample 

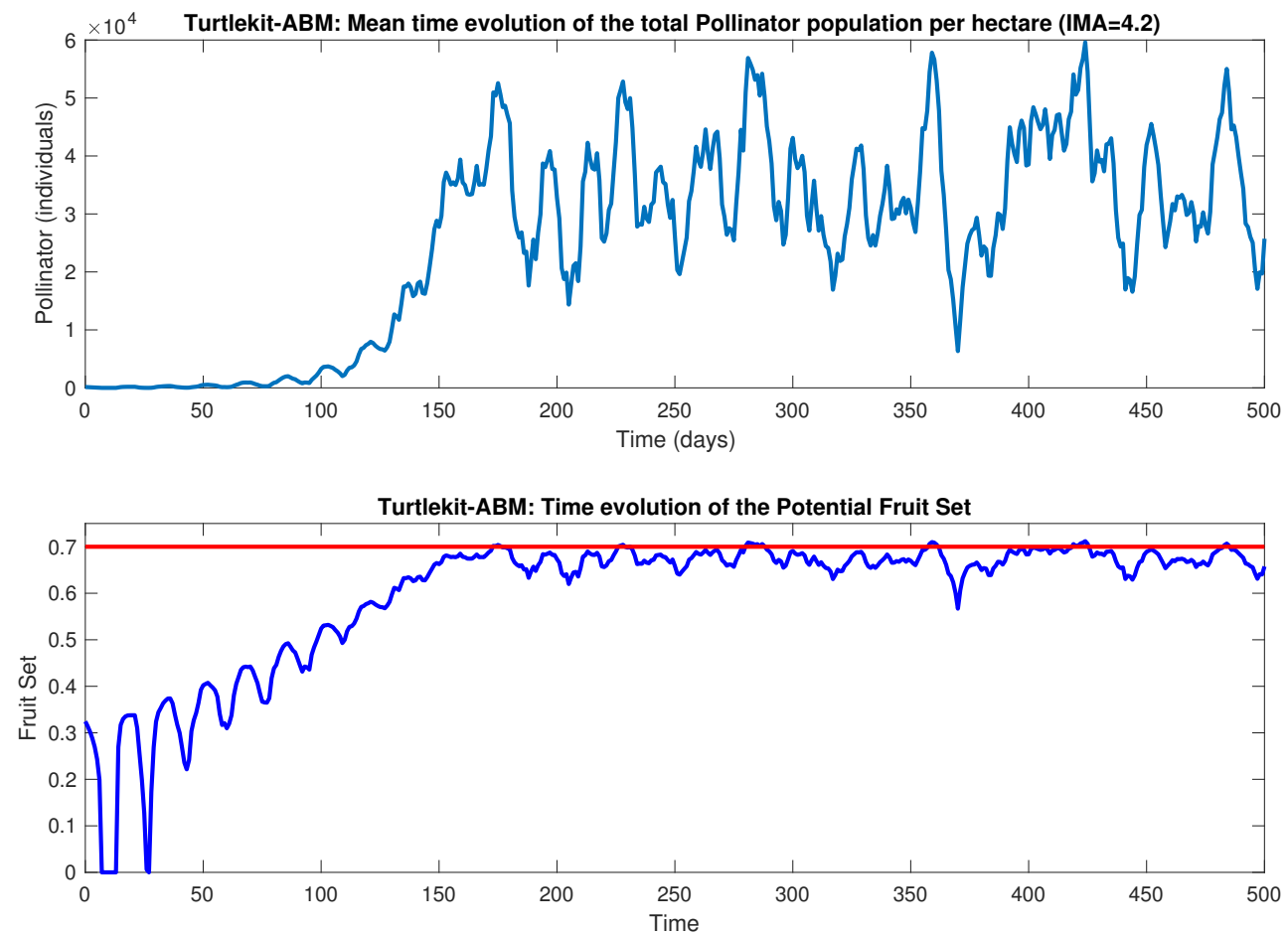

FIGURE 11 ABM simulations. Time evolution of (a) the pollinator population; (b) the potential fruit set when the MIA is 4.2

size. So, the ABM parameters we tested and their bounds, i.e minimum and maximum values, are given below, in Table 1 page 17

\begin{tabular}{l|c|c|c} 
Parameter & Acronym & Min value & Max value \\
\hline & & & \\
Time Between Inflorescences in Anthesis & TBIA & 9 & 18 \\
Male Inflorescence Ratio & MIR & 0.01 & 0.15 \\
Palm Larvae Capacity $\beta$ & PLC & 5000 & 20000 \\
Male Inflorescence Attraction Radius $r_{\text {mas }}$ & MIAR & 2 & 30 \\
Larva Maturation Time (days) & LMT & 10 & 20 \\
Larvae mortality percentage & LM & 0.03 & 0.09 \\
Larvae Per day per Female & LPF & 1 & 6 \\
Larvae Female Ratio & LFR & 0.6 & 0.8 \\
Pollinator Longevity (days) & PL & 5 & 10 \\
Pollinator Speed (m/h) & PS & 16 & 48
\end{tabular}

TABLE 1 Bounds used for the extended-FAST sensibility analysis of the ABM

Using these bounds, we set a FAST sample size of 256 as input for generating the experimental plan required for the analysis, which leaded to a plan composed of 2560 simulations, for each of which we consider two model responses: 
The averaged populations of (1) adults and (2) larvae. For being able to carry out this analysis, we used a 25 Xeon CPUs cluster with $256 \mathrm{~Gb}$ of RAM.

Fig. 12 page 18, and Fig. 13 , page 19, show the results obtained using the previous experimental settings using FAST. From this sensibility analysis, we can draw several conclusions considering different perspectives as now discussed.

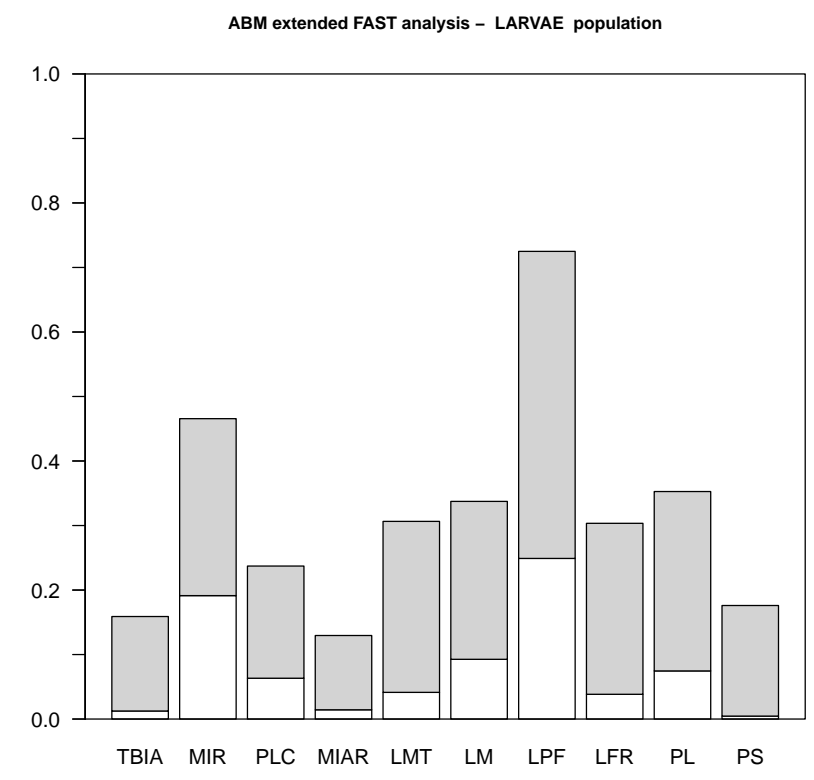

FIGURE 12 Sensitivity analysis against the larvae population dynamics

Firstly, about the ABM itself, one can see that all the parameters have some influence on the outputs, which suggests that we can keep on working on researching their most relevant values with respect to their biological reality.

Secondly, considering the qualitative aspect of the results, one can see that LPF, LM, LMT, MIR and PL are the most influential parameters for now. This is coherent with the fact that those biological parameters are intuitively crucial with respect to the population dynamics. For instance, it comes as no surprise that the number of Larvae Per Female (LPF) greatly influences the resulting populations. So, this analysis gives us some guidelines for future research (data acquisition) on the field. Especially, as LPF seems to be the most critical parameter, it is therefore crucial to validate this parameter using field observations since very different values can be found in the literature. So, the same remark holds for the Male Inflorescence Ratio (MIR), the larvae mortality and their maturation time (respectively LM and LMT) and the adult weevils life expectancy (PL).

Finally, the fact that the Male Inflorescence Attraction Radius (MIAR) and the pollinator speed parameters do not play a significant role on their own gives us some clues about how we can improve the individual behaviors modeling. Indeed, this suggests that how the weevils move and explore their environment could be revised so that they have more significant impact as it is surely the case in the field reality. For now, all the modeled weevils have equivalent behaviors from a qualitative point of view. In addition, we implicitely assume that the "plume" of attraction of each male inflorescence is a disk of radius MIAR, while it is known that this can be very complex, in particular when wind occurs under the canopy, spreading anis odors on a wide range. This may certainly change the behaviour of the weevils and thus impact their dispersal. 


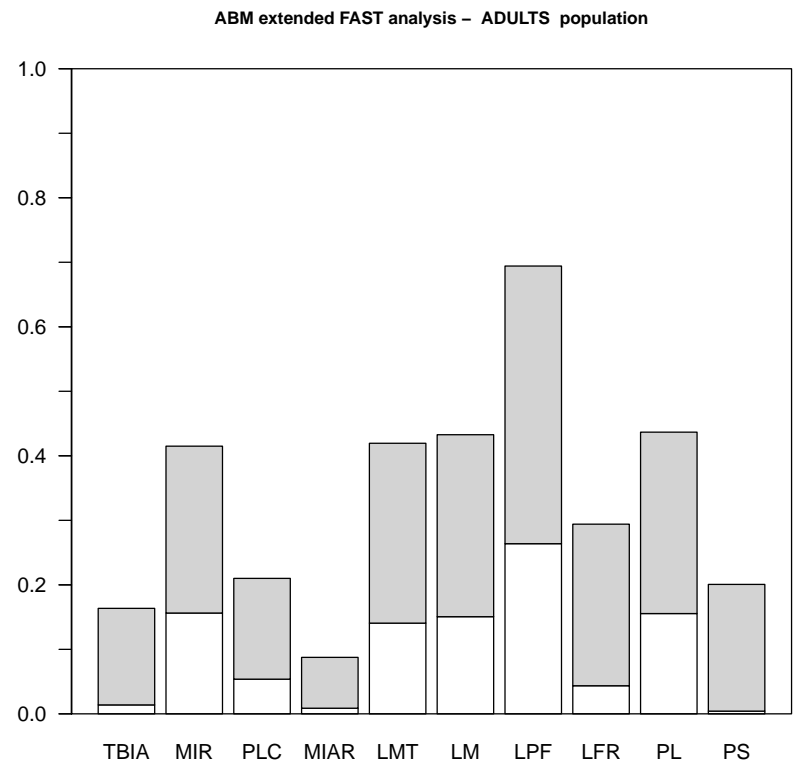

FIGURE 13 Sensitivity analysis against the adults population dynamics

\section{5 | CONCLUSION}

This work is a first step towards the complete modeling of oil palm-pollinator interactions, that is a very complex systems for which we have only a very partial knowledge. Surprisingly, from the agronomic point of view, if oil palm has been very well studied and various practices developed in different places around the world, little is known about Elaeidobius sp. and other pollinators and their interactions with palm trees. Syed and collaborators have done an amazing work in the early 80's leading to the introduction of E. kameirunicus in Malaysia, however since then, only some "sporadic" field works, which are not always useful from the modeling point of view, have been conducted. However, modeling is a powerful tool to test assumptions and/or think on real experiments to validate or refute these assumptions. Since we have only a partial knowledge on the whole system, mathematical modeling, through ODEs, is powerful in that sense that we do not need to have detailed system knowledge. Conversely, an ABM approach needs deeper knowledge to realistically implement and simulate the system. Moreover, patchy models simulations are fast, while the ABM is more labour intensive due to multiple individual behavior computations. This, of course, makes the ABM simulations more "realistic" and allows to study other aspects of this complex system.

However, even at this preliminary modeling stage, we would like to emphasize that both approaches are highly complementary. Complementary in that sense that both models can be helpful to determine the important parameters or missing knowledge on which one has to focus on and/or prioritize.

Based on this first modeling step, and our preliminary results, we intend to go further in terms of experiments, modeling, analysis, and simulations. Clearly, field experiments are needed to increase our knowledge about the weevils and their interactions with the inflorescences. This additional knowledge are particularly important for the ABM approach that is only based on simulations (in the sense of all aspects of ABM cannot be theoretically analyzed) and of course, they will be useful to improve the patchy model too, as well as to better estimate the parameters.

Last but not least, additional experiments on the relationship between the fruit set and the weevils population size per ha (or per inflorescence) should be updated. Indeed, since the 80 's, new palm trees varieties have been selected leading, probably, to changes within their relationship with the weevils population (positively or negatively). 
From the modeling point of view, our models can be improved or complexified taking into account the role of female inflorescences on the pollinator dispersal, and the fruit set.

Acknowledgments. The first author thanks PalmElit and CIRAD for financial support and fruitfull discussions.

\section{ANNEXE A: BIOLOGICAL PARAMETERS OF ELAEIDOBIUS KAMERUNICUS}

According to the literature $\frac{8716}{}$ and field experts knowledge, we have considered the following values for the model parameters

\begin{tabular}{l|l} 
Parameters & Mean value \\
\hline & \\
Adult death rate, $\mu_{P}$ & $1 / 7\left(\right.$ day $\left.^{-1}\right)$ \\
Larvae death rate $\mu_{L}$ & $0.05\left(\right.$ day $\left.^{-1}\right)$ \\
Larvae full development, $1 / \nu$ & $14($ day $)$ \\
spreading ability, $\lambda$ & $20,10,20 / 3$ (estimated) \\
sex-ratio, $r$ & $2 / 3$ \\
Newborns per Female, $b$ & $3\left(\right.$ day $\left.^{-1}\right)$ \\
Max. Larvae Capacity, $\beta$ & $10^{4}$ \\
\hline & \\
Pollinator speed, $v_{M A S}$ & $20(\mathrm{~m} / \mathrm{h})$ \\
MIA - radius of attraction, $r_{M A S}$ & $25(\mathrm{~m})$
\end{tabular}

TABLE 2 E. kamerunicus parameters

\section{References}

1. Corley R.H.V., Tinker P.B. The Oil Palm. John Wiley \& Sons, Ltd; 2015.

2. Syed R.A. Studies on oil palm pollination by insects. Bulletin of Entomological Research. 1979;69(2):213-224.

3. Syed R.A. Insect pollination of oil palm: feasibility of introducing Elaeidobius spp. into Malaysia. In: :263289Incorporated Society of Planters; 1982.

4. Syed R.A., Law I.H., Corley R.H.V. Insect pollination of oil palm: introduction, establishment and pollinating efficiency of Elaeidobius kamerunicus in Malaysia. Planter. 1982;58(681):547-561.

5. Appiah S.O., Agyei D. Studies on Entomophil pollination towards sustainable production and increased profitability in the oil palm: a review. Elixir Agriculture. 2013;55:12878-12883.

6. Tuo Y., Koua H.K., Hala N. Biology of Elaeidobius kamerunicus and Elaeidobius plagiatus (Coleoptera: Curculionidae), main pollinators of oil palm in West Africa. European Journal of Scientific Research. 2011;49(3):426-432.

7. Kevan P.G., Hussein M.Y., Hussey N., Wahid M.B. Modelling the use of Elaeidobius kamerunicus for pollination of oil palm.. Planter. 1986;62:89-99. 
8. Dhileepan K. Variation in populations of the introduced pollinating weevil (Elaeidobius kamerunicus)(Coleoptera: Curculionidae) and its impact on fruitset of oil palm (Elaeis guineensis) in India. Bulletin of entomological research. 1994;84(4):477-485.

9. Dhileepan K. Pollen carrying capacity, pollen load and pollen transferring ability of the oil palm pollinating weevil Elaeidobius kamerunicus Faust in India. Oleagineux (Paris). 1992;47(2):55-61.

10. Wahid M.B., Kamarudin N.H.J. Role and effectiveness of Elaeidobius kamerunicus, Thrips hawaiiensis and Pyroderces sp. in pollination of mature oil palm in peninsular Malaysia. Elaeis. 1997;9(1):1-16.

11. Kouakou M., N'Klo H., Akpesse A.A.M., et al. Comparative efficacy of Elaeidobius kamerunicus, E. plagiatus, E. subvittatus (Coleoptera: Curculionidae) and Microporum spp.(Coleoptera: Nitidulidae) in the Pollination of Oil Palm (Elaeis guineensis). Journal of Experimental Biology. 2014;2:6.

12. Woittiez L.S., Wijk M.T., Slingerland M., Noordwijk M., Giller K.E. Yield gaps in oil palm: A quantitative review of contributing factors. European journal of agronomy. 2017;83:57-77.

13. Jacquemard J.-C. Le palmier à huile. Editions Quae; 2012.

14. Dumont Y., Thuilliez J. Human behaviors: A threat to mosquito control?. Mathematical biosciences. 2016;281:9-23.

15. Hanski I. Metapopulation Ecology. University Press Oxford; 1999.

16. Bowong S., Dumont Y., Tewa J.J. A patchy model for chikungunya-like diseases. Biomath. 2013;2(1):1307237.

17. Van Dyke Parunak H., Savit Robert, Riolo RickL. Agent-Based Modeling vs. Equation-Based Modeling: A Case Study and Users' Guide. In: Sichman J.S., Conte R., Gilbert N, eds. Multi-Agent Systems and Agent-Based Simulation, Lecture Notes in Computer Science, vol. 1534: Springer Berlin Heidelberg 1998 (pp. 10-25).

18. Michel F., Ferber J., Drogoul A. Multi-Agent Systems and Simulation: a Survey From the Agents Community's Perspective. In: Uhrmacher Adelinde, Weyns Danny, eds. Multi-Agent Systems: Simulation and Applications, Computational Analysis, Synthesis, and Design of Dynamic Systems. CRC Press - Taylor \& Francis 2009 (pp. $3-52)$.

19. Michel F., Beurier G., Ferber J. The TurtleKit Simulation Platform: Application to Complex Systems. In: Akono Alain, Tonyé Emmanuel, Dipanda Albert, Yétongnon Kokou, eds. Workshops Sessions of the Proceedings of the $1^{\text {st }}$ International Conference on Signal-Image Technology and Internet-Based Systems, SITIS 2005, November 27 December 1, 2005, Yaoundé, Cameroon, :122-128IEEE; 2005.

20. Sklar E. NetLogo, a Multi-agent Simulation Environment. Artificial Life. 2007;13(3):303-311.

21. Resnick M. Turtles, termites, and traffic jams: Explorations in massively parallel microworlds. MIT Press; 1994.

22. Hermellin E., Michel F. GPU Delegation: Toward a Generic Approach for Developping MABS using GPU Programming. In: Jonker Catholijn M., Marsella Stacy, Thangarajah John, Tuyls Karl, eds. Proceedings of the 2016 International Conference on Autonomous Agents \& Multiagent Systems, Singapore, May 9-13, 2016, :1249-1258; 2016.

23. Ferber J., Gutknecht O., Michel F. From Agents to Organizations: an Organizational View of Multi-Agent Systems. In: Giorgini Paolo, Müller Jörg P., Odell James, eds. Agent-Oriented Software Engineering IV, Lecture Notes in Computer Science, vol. 2935: Springer Berlin Heidelberg 2004 (pp. 214-230). 
24. Syed R.A., Salleh A. Population of Elaeidobius kamerunicus Fst. in relation to fruitset. In: Institut Penyelidikan Minyak Kelapa Sawit Malaysia Selangor (Malaysia) Kuala Lumpur, ed. International Oil Palm/Palm Oil Conferences Progress and Prospects Conference 1: Agriculture, :528-534; 1987.

25. Marino S., Hogue I.B., Ray C.J., Kirschner D.E. A methodology for performing global uncertainty and sensitivity analysis in systems biology. Journal of theoretical biology. 2008;254(1):178-196. 